\subsection{Schlußfolgerungen}

Vor dem Hintergrund der hier beschriebenen, für die Aale optimalen Hälterungsbedingungen, sind die Ergebnisse der Studie besonders aussagekräftig. Die Auswahl der untersuchten Organohalogene erfolgte auf der Basis ihrer ökologischen und toxikologischen Bedeutung; sie deckt sich weitgehend mit den Untersuchungsparametern der Hessischen Landwirtschaftlichen Versuchsanstalt bei der Überprüfung der Schadstoffbelastung von Lahnfischen (HLVA 1990). In der eigenen Studie wurde statt des Lindans ( $\gamma$ $\mathrm{HCH}$ ) das $\alpha$-Isomere wegen seiner vergleichsweise höheren Persistenz in der Umwelt ausgewählt (DFG 1983).

\section{Danksagung}

Die Verfasser danken der Sportfischer-Vereinigung Marburg und Umgebung e.V. für die Unterstützung bei der Durchführung der Studie.

\section{Literatur}

BECK, H.; W. MATHAR: Analysenverfahren zur Bestimmung von ausgewählten PCB-Einzelkomponenten in Lebensmitteln. Bundesgesundheitsblatt 28, 1-12 (1985)

BoISELle, C.; G. HILDEBRANDT: Versuche zur ClKW-Dekontamination bei Aalen. Arch. Lebensmittelhyg. 40, 1-24 (1989)
Bruggeman, W.A.; A. Opperhuizen; A. Wijbenga; O. Hutzinger: Bioaccumulation of super-lipophilic chemicals in fish. Toxicol. Environmental Chem. 7, 173-189 (1984)

DFG 1983: Hexachlorcyclohexan als Schadstoff in Lebensmitteln. Deutsche Forschungsgemeinschaft, Forschungsbericht, Verlag Chemie, Weinheim 1983

HLVA 1990: Hessische Landwirtschaftliche Versuchsanstalt, Auswertung der Untersuchungsergebnisse von Lahnfischen, gefangen im Jahre 1989. Wiesbaden 1990

Koss, G.; S. Seubert; A. Seubert; J. Seidel; W. Koransky; H. IPPEN: Studies on the toxicology of hexachlorobenzene. V. Different phases of porphyria during and after treatment. Arch. Toxicol. 52, $13-22(1983)$

Koss, G.: 2,2',3,4,4',5,5'-Heptachlorbiphenyl. Manuskript in Vorbereitung

ManN, H.: Untersuchung über die Hälterung von Aalen. Arch. Fischereiwiss. 11, $145-151$ (1960)

PHmV 1989: Verordnung über Höchstmengen an Pflanzenschutz- und sonstigen Mitteln sowie anderen Schädlingsbekämpfungsmitteln in oder auf Lebensmitteln und Tabakerzeugnissen (Pflanzenschutzmittel-Höchstmengenverordnung - PHmV) vom 16. Okt. 1989, BGBl., 1989, Teil I, S. $1861-1908$

SHmV 1988: Verordnung über Höchstmengen an Schadstoffen in Lebensmitteln (Schadstoff-Höchstmengenverordnung - SHmV) vom 23. März 1988. BGBI., 1988, Teil I, S. $422-424$

Tesch, F.-W.: Der Aal. Biologie und Fischerei. Verlag Paul Parey, Hamburg/Berlin 1983, pp. 20

\title{
Abbau von Linearen Alkylbenzolsulfonaten (LAS) unter Labor- und Freilandbedingungen mit einer neuen HPLC-Bestimmungsmethode
}

\section{- Abschätzung des LAS-Gefährdungspotentials}

\section{J. Pflugmacher}

Dr. J. Pflugmacher, Biologische Bundesanstalt für Land- und Forstwirtschaft, Institut für Chemikalienprüfung, Königin-Luise-Straße 19, D-W-1000 Berlin 33

Zusammenfassung. Das Abbauverhalten von Linearen Alkylbenzolsulfonaten (LAS) im Boden wurde im Labor und im Freiland unter verschiedenen Bedingungen mit einer neu entwickelten Aufreinigungs- und Bestimmungsmethode auf Basis der HPLC untersucht, mit der sich Rückstände von LAS im Boden und Klärschlamm bis zu einer Nachweisgrenze von $0,01 \mathrm{mg} / \mathrm{kg}$ erfassen lassen. Die Wiederfindungsrate bei Zusatzversuchen im Boden betrug bei einer Konzentration von $0,02 \mathrm{mg} / \mathrm{kg} 90,8 \pm 5,4 \%$. Unter aeroben nichtsterilen Bedingungen verläuft der Abbau am schnellsten (Halbwertzeiten: 5 - 7 Tage), unter anaeroben/anaeroben sterilen Bedingungen wesentlich langsamer (Halbwertzeiten: $40-120$ Tage).

\section{Abstract.}

Degradation of Linear Alkylbenzenesulfonates (LAS) in Soil Under Laboratory and Field Conditions with a New Detection Method. The degradation of linear alkylbenzenesulfonates (LAS) in soil was investigated under different laboratory and field conditions using a new HPLC method. The average recovery of LAS on a concentration level of $0.02 \mathrm{mg} / \mathrm{kg}$ in soil was $90.8 \pm 5.42 \%$. Detection limit $(S / N \geq 3)$ for the matrices soil and sewage sludge was $0.01 \mathrm{mg} / \mathrm{kg}$. Under aerobic non sterile conditions LAS decline rapidly (half lives: 5-7 days); under anaerobic/anaerobic sterile conditions half lives were $40-120$ days.

\section{Einleitung und Problemstellung}

Lineare Alkylbenzolsulfonate (LAS) sind z.Zt. die am meisten produzierten anionischen oberflächenaktiven Substanzen. Sie werden als Bestandteil von Waschmitteln eingesetzt und gehören wegen ihres hohen Produktionsvolumens zu den mengenmäßig bedeutendsten organischen Chemikalien, die in Abwässern auftreten. Bei der Abwasserreinigung in den Klärwerken erfolgt eine Anreicherung im Klärschlamm (Durchschnitt $4 \mathrm{~g} / \mathrm{kg}$ Trockensubstanz nach [4]. Mit der Ausbringung von Klärschlamm auf landwirtschaftliche Nutzflächen gelangen sie in den Boden. Während über das Verhalten und die Abbaubarkeit im aquatischen Bereich vielfach publiziert wurde, sind für den terrestrischen Bereich nur wenige Daten verfügbar [10]. Kenntnisse über das Abbauverhalten im Boden sind wichtig, zum einen wegen der hohen Eintragsmenge, zum anderen wegen ihrer oberflächenaktiven Eigenschaft, die Löslichkeit anderer schwer wasserlöslicher Verbindungen zu erhöhen [12]. 
Nach den von SwISHER [11] gesammelten Adsorptionsdaten werden anionische Tenside nicht nennenswert sorbiert. Daher können nicht abgebaute Tenside in das Grundwasser gelangen und aufrund ihres Leachingverhaltens auch die Rückstände von Pflanzenschutzmitteln oder anderer organischer Umweltchemikalien mobilisieren, wie Laboruntersuchungen belegen. Als Grundlage für die Abschätzung dieses Gefährdungspotentials $[5,6]$ war es notwendig, den Abbau von LAS zu untersuchen.

Zur Bestimmung von LAS im aquatischen Milieu sind eine Reihe von Methoden auf der Basis Kolorimetrie, Gaschromatographie, Gaschromatographie-Massenspektrometrie, Atomabsorptionsspektrometrie und Hochdruckflüssigkeitschromatographie bekannt.

Für die Bestimmung von LAS in Böden oder Sedimenten sind dem Autor nur wenige Arbeiten bekannt [1, 2, 7]. Die Methode von TreHY et al. [7] zur Bestimmung in Sedimenten ist apparativ sehr aufwendig, weil die Bestimmung mit Hilfe der GC-MS erfolgt und weist eine relativ geringe Empfindlichkeit von $0,5 \mathrm{mg} / \mathrm{kg}$ Sediment auf.

Die flüssigkeitschromatographischen Bestimmungsmethoden $[1,2]$ basieren auf der Arbeit von NAKAE et al. [8], die LAS mit Hilfe der HPLC und anschließender Fluoreszenzdetektion in Flußwasser ohne vorherige Aufreinigung und Anreicherung bestimmte. Sie beinhalten entweder aufwendige Reinigungsschritte durch zweifache Festphasenextraktion [1] oder eine komplizierte HPLC-Bestimmungsmethode [2].

\section{Material und Methoden}

\subsection{Material}

Versuchschemikalie (LAS): Marlon A $350(50 \%) \mathrm{C}_{10}-\mathrm{C}_{13}$-LASGemisch (Fa. Hüls)

Proben: Testböden der Landesuntersuchungs- und Forschungsanstalt Speyer (Bodendaten $\rightarrow$ Tabelle 1)

Tabelle 1: Analysedaten der getesteten Böden

\begin{tabular}{l|c|c|c}
\hline $\begin{array}{l}\text { Testböden der } \\
\text { Lufa Speyer }\end{array}$ & 2.1 & 2.2 & 2.3 \\
\hline org. C (\%) & 0.90 & 2.06 & 1.01 \\
\hline Schluff (\%) & 60.8 & 5.3 & 46.1 \\
\hline pH-Wert & 5.7 & 6.0 & 5.7 \\
\hline Ton (\%) & 1.9 & 4.3 & 6.0 \\
\hline
\end{tabular}

\begin{tabular}{lr|c|c|c}
\hline $\begin{array}{l}\text { Dahlemer } \\
\text { Versuchsfeld }\end{array}$ & & & \\
\hline \multicolumn{2}{l|}{\begin{tabular}{l} 
Schlagbez. \\
\hline org. C $\quad 21$
\end{tabular}} & 251 & $27 \mathrm{C}$ \\
\hline Schluff & 1.21 & 0.75 & 0.58 \\
\hline $\mathrm{pH}$ & 14.5 & 22.6 & 10.2 \\
\hline \multicolumn{2}{l|}{ Ton } & 6.2 & 6.3 & 6.7 \\
\hline
\end{tabular}

Boden des Versuchsfeldes der Biologischen Bundesanstalt BerlinDahlem (Bodendaten $\rightarrow$ Tabelle 1)

Klärschlammproben des Klärwerkes Berlin-Mariendorf: Die Klärschlammproben wurden sofort nach Probenahme mit $1 \%$ Formalin (37\% Formaldehyd) konserviert.

Sterilisation: Die Sterilisation erfolgte durch $20 \mathrm{~min}$ autoklavieren bei einer Temperatur von $120^{\circ} \mathrm{C}$ und einem Druck von 1,6 bar.

\subsection{Versuchsdurchführung und Methoden}

Bei der vorgestellten Methode wird die Bodenprobe nach der Extraktion durch Festphasenextraktion an einer Anionenaustauschersäule gereinigt. Die anschließende Trennung erfolgt mit Hilfe der HPLC durch Ionenpaarchromatographie mit einem Eluenten aus Wasser/Methanol unter Zusatz von Tetrabutylammoniumhydrogensulfat mit anschließender Bestimmung durch UV-Adsorption oder mit Hilfe der Fluoreszensmessung.

\section{Versuchsdurchführung}

Die Abbauversuche wurden jeweils mit $1 \mathrm{~kg}$ (Bezugsbasis: Trocknung im Ofen bei $105^{\circ} \mathrm{C}, 24 \mathrm{~h}$ ) luftgetrockneter Versuchserde bei einer Bodenfeuchte von $20 \%$ der max. Wasserkapazität durchgeführt. Die Kontamination des Bodens auf eine Anfangskonzentration von $100 \mathrm{mg} / \mathrm{kg}$ wurde in der Weise vorgenommen, daß die berechnete Menge LAS als wäßrige Lösung auf den Boden gegeben und anschlieBend durch Zugabe von Wasser die gewünschte Bodenfeuchte von $20 \%$ eingestellt wurde. Zur homogenen Verteilung der Chemikalie wurde der Boden dann $30 \mathrm{~min}$ in einer Mischmaschine gerührt und schließlich in die als Versuchsgefäße dienenden Bechergläser überführt. Für die Versuchsansätze unter anaeroben Bedingungen wurden die Versuchsgefäße in einen Exsikator gestellt und nach Evakuierung unter einer Stickstoffatmosphäre gehalten.

Die Kontamination der Böden im Freilandversuch auf eine Anfangskonzentration von $100 \mathrm{mg} / \mathrm{kg}$ erfolgte durch Lösen der entsprechenden Aufwandmenge in Wasser und Ausbringen mit einer Pflanzenschutzspritze. Der Berechnung der Aufwandmenge wurde eine Bodenschicht von $5 \mathrm{~cm}$ mit einer (mittleren) Dichte von $1,5 \mathrm{~g} / \mathrm{m}^{2}$ zugrunde gelegt.

\section{Extraktion und Aufreinigung der Bodenprobe}

$20 \mathrm{~g}$ der Bodenprobe werden mit $70 \mathrm{ml}$ Methanol $5 \mathrm{~h}$ im Soxhlet-Extraktor extrahiert. Anschließend wird der Methanolextrakt mit Hilfe eines Rotationsverdampfers bei einer Temperatur von $45^{\circ} \mathrm{C}$ zur Trockne eingeengt. Der Rückstand wird mit $2 \mathrm{ml}$ bidestilliertem Wasser aufgenommen.

\section{Extraktion von Klärschlamm}

Zunächst wird die Probe 5 min geschüttelt, um eine gleichmäßige Verteilung des Feststoffes zu erreichen. Sodann werden $20 \mathrm{ml}$ Klärschlamm (80,8\% Wassergehalt) mit $250 \mathrm{ml}$ Methanol $5 \mathrm{~h}$ an einer Soxhlet-Apparatur extrahiert. Der Soxhlet-Extrakt wird über ein Blauband-Filter abgenutscht und das Filtrat im Rotationsverdampfer zur Trockne eingeengt. Der Rückstand wird anschließend in destilliertem Wasser aufgenommen. Die weitere Aufarbeitung erfolgt wie bei den Bodenproben. 


\section{Aufreinigung}

Anschließend erfolgt die Reinigung an einer Anionenaustauschersäule, die vorher auf folgende Weise konditioniert wird:

Die Säule wird völlig mit $n$-Hexan gefüllt und das Hexan unter Vakuum durch die Säule gesaugt. Anschließend wird 1 min Luft durch die Säule gesaugt. Sodann wird die Säule zweimal mit Methanol und anschließend mit Wasser gefüllt und das Methanol bis zur Oberfläche des Sorbens abgesaugt, wobei darauf zu achten ist, daß die Säule nicht trocken läuft. Sodann wird der Probenextrakt auf die Säule gegeben und unter Vakuum langsam auf das Sorbens gezogen. Dann wird die Säule zweimal vollständig mit Methanol gefüllt und abgesaugt. Das Eluat wird verworfen.

Das LAS wird durch Aufgabe von $10 \mathrm{ml}$ mit Hilfe von $\mathrm{HCl}$ auf $\mathrm{pH} 2$ angesäuertem Methanol eluiert und wiederum zur Trockne eingeengt. Die Probe wird dann mit der mobilen Phase des zur Trennung verwendeten Eluenten aufgenommen und nach Konzentration auf ein Volumen von 0,5 $1 \mathrm{ml}$ gebracht.

\section{Methoden}

\section{Chromatographische Analyse}

Die Methodenentwicklung bzw. die Analysen wurden mit einem Hewlett Packard 1050 Flüssigkeitschromatographen, ausgerüstet mit einem automatischen Probengeber und einem UV-Spektralphotometer, sowie dem Fluoreszenzdetektor RF-S 35 der Fa. Shimadzu, durchgeführt. Die Integration der Daten erfolgte mit den Hewlett Packard Datensystem HPLC-Chem-Station bzw. im Falle der Fluoreszenzmessung mit einem Integrator Modell 4270 der Fa. Spectra Physics. Bei dem UV-Detektor betrug die Detektionswellenlänge $228 \mathrm{~nm}$, während bei dem Fluoreszenzdetektor die Anregungswellenlänge $225 \mathrm{~nm}$ und die Emissionswellenlänge $285 \mathrm{~nm}$ betrug. Die chromatographische Trennung wurde mit Hilfe der Ionenpaarchromatographie auf einer $5 \mu \mathrm{m}$ Lichrospher RP-8-Säule ( $25 \mathrm{~cm} \times 4 \mathrm{~mm}$ i.D.), welche mit einer $3 \mathrm{~cm}$ Vorsäule mit identischem Füllungsmaterial gekoppelt war, vorgenommen.

Als mobile Phase wurde ein binäres Gemisch ciner $0,01 \mathrm{~m}$ Lösung von Tetrabutylammoniumhydrogensulfat mit einem eingestellten $\mathrm{pH}$ von 2.30 und Methanol benutzt. Die Trennung wurde unter isokratischen Bedingungen mit einem Gemisch von $20 \%$ Salzlösung und $80 \%$ Methanol bei einer Flußrate von $1.2 \mathrm{ml} / \mathrm{min}$ durchgeführt.

\section{Quantifizierung}

Die Quantifizierung des LAS-Gehaltes und der Anteil der einzelnen LAS-Homologen des für die Versuche eingesetzten Handelspräparates Marlon A 350 erfolgte durch Messung gegen die Standardsubstanz Natriumdodecylbenzolsulfonat. Da der Standard neben den $\mathrm{C}_{12}$-LAS Homologen

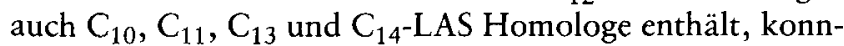
ten durch Vergleich der Retentionszeiten die Anteile der Hauptkomponenten im Marlon A 350 bestimmt werden, für die sich folgende Werte ergaben:

$\mathrm{C}_{10}(7,3 \%), \mathrm{C}_{11}(0,05 \%), \mathrm{C}_{12}(34,5 \%), \mathrm{C}_{13}(5,63 \%)$. Als Referenzstandard wurde der Peak der mengenmäßig bedeutendsten Komponente des $\mathrm{C}_{11}$-LAS Homologen be- nutzt. Die Wiederfindungsrate bei Zusatzversuchen im Boden betrug bei einer Konzentration von $0,02 \mathrm{mg} / \mathrm{kg}$ $90,8 \pm 5,4 \%$.

Die Empfindlichkeit für die UV-Messung beträgt bei einem Signal-Rauschverhältnis von 3:1 25 ng und bei der Fluoreszenzmessung $2 \mathrm{ng}$. Für die Matrices Boden und Klärschlamm beträgt die Nachweisgrenze für LAS bei UV-Detektion $0,05 \mathrm{mg} / \mathrm{kg}$ und bei der Fluoreszenzdetektion $0,01 \mathrm{mg} / \mathrm{kg}$. Eine Steigerung der Nachweisempfindlichkeit durch Erhöhung des Probengewichtes bei gleichzeitiger Verwendung von Austauschersäulen höherer Kapazität erscheint möglich.

\section{Ergebnisse und Diskussion}

\subsection{Laborbedingungen}

In Tabelle 2 sind die Halbwertszeiten für den Abbau von LAS im Labor unter verschiedenen Bedingungen in Standardböden der Lufa-Speyer aufgelistet. Bei der Ermittlung der Halbwertszeiten wurde davon ausgegangen, daß der Abbau nach der Kinetik einer Reaktion erster Ordnung stattfindet $(\rightarrow$ Tabelle 2$)$.

Tabelle 2: Die Halbwertszeiten für den Abbau von LAS unter verschiedenen Bedingungen im Labor in Standardböden der Lufa-Speyer

\begin{tabular}{l|c|c|c}
\hline \multirow{2}{*}{$\begin{array}{l}T_{1 / 2} \\
\text { (Tage) }\end{array}$} & 2.1 & 2.2 & 2.3 \\
\cline { 2 - 4 } $\begin{array}{l}\text { aerob } \\
\text { unsteril }\end{array}$ & 7.0 & 6.5 & 6.5 \\
\hline $\begin{array}{l}\text { anaerob } \\
\text { unsteril }\end{array}$ & 8.5 & - & 5.9 \\
\hline $\begin{array}{l}\text { aerob } \\
\text { steril }\end{array}$ & 42.2 & 36.4 & 48.4 \\
\hline $\begin{array}{l}\text { anaerob } \\
\text { steril }\end{array}$ & 64.4 & 119.2 & 122.0 \\
\hline
\end{tabular}

Der Tabelle läßt sich entnehmen, daß der Abbau, wie zu erwarten war, im nicht sterilisierten Boden unter aeroben $\mathrm{Be}$ dingungen am schnellsten vor sich geht.

Die Differenzen im Abbau zwischen aeroben und anaeroben Bedingungen sind überraschenderweise nur gering bzw. nicht vorhanden, obwohl der anaerobe Abbau thermodynamisch nicht so begünstigt abläuft. Auch die Unterschiede der Halbwertszeiten in den verschiedenen Testböden sind nur gering, was darauf hindeutet, daß keine nennenswerte Adsorption stattfindet, da sonst der Abbau verzögert wäre.

In sterilisiertem Boden unter aeroben Bedingungen erfolgt der Abbau wesentlich langsamer, die entsprechenden Halbwertszeiten bewegen sich im Bereich von 36-48 Tagen. Unter sterilen anaeroben Bedingungen ergibt sich eine nochmalige Verlangsamung des Abbaus, je nach Versuchsboden mit Halbwetszeiten von 64-122 Tagen, wobei eine deutliche Differenz zwischen dem Boden 2.1 mit T 1/2 = 
64 Tagen und den anderen Testböden 2.2 und 2.3 mit $\mathrm{T} 1 / 2=119-122$ Tagen besteht. Aus den Bodendaten ist nicht ableitbar, wodurch die Unterschiede in der Abbauleistung zustande kommen, zumal unter den anderen Versuchsbedingungen keine so deutliche Differenzierung zu erkennen war. Ein Vergleich der unter den verschiedenen Bedingungen ermittelten Halbwertszeiten macht deutlich, daß der Abbau von LAS im Boden hauptsächlich von den beiden Faktoren mikrobielle Aktivität und Sauerstoff abhängig ist, wobei die mikrobielle Aktivität den größten Einfluß auf den Abbau hat.

\subsection{Freilandbedingungen}

In Tabelle 3 sind die Halbwertszeiten von LAS im Freiland auf verschiedenen Standorten mit den o.g. Bodenbedingungen unter verschiedener Vegetation aufgeführt $(\rightarrow$ Tabelle 3). Die Halbwertszeiten liegen sämtlich in einem engen Bereich von 5-7 Tagen. Unterschiedlicher Boden und Bewuchs haben offensichtlich keinen meßbaren Einlfuß auf die Abbaurate. Der Vergleich mit den unter nicht sterilen aeroben Bedingungen im Labor erzielten Abbaudaten zeigt geringfügig kürzere Halbwertszeiten für das Freiland.

Tabelle 3: Die Halbwertszeiten des Abbaus von LAS im Freiland unter verschiedenen Bewuchsbedingungen

\begin{tabular}{l|c|c|c}
\hline \multirow{2}{*}{$\begin{array}{l}\text { T 1/2 } \\
\text { (Tage) }\end{array}$} & \multicolumn{3}{|c}{ Bodentyp } \\
\cline { 2 - 4 } & Sandboden & lehm.Sandboden & Parabraunerde \\
\hline Hafer & 5.3 & 5.4 & 6.4 \\
\hline Brache & 6.7 & 5.3 & 6.0 \\
\hline $\begin{array}{l}\text { Mischbe- } \\
\text { wuchs }^{\mathrm{a}}\end{array}$ & 6.9 & 6.4 & 7.4 \\
\hline
\end{tabular}

${ }^{a}$ Mischbewuchs: Mischung aus Avena sativa (Hafer), Lolium perenne (Weidelgras) und Chenopodium album (Weißer Gänsefuß) im Verhältnis $50: 25: 25$

Die dem Autor zugänglichen zwei Publikationen $[9,10]$ über den Abbau von LAS unter Freilandbedingungen berichten von Halbwertszeiten von 8-22 Tagen, wobei die Bestimmungen allerdings unspezifisch durch kolorimetrische Messung eines Farbkomplexes mit Methylenblau erfolgten.

\subsection{Verleich von Labor- und Freilandbedingungen}

Aus den Ergebnissen der Labor- und Freilandversuche für den aeroben unsterilen Abbau kann abgeleitet werden, daß LAS bei normaler mikrobieller Aktivität und ausreichender Belüftung vergleichbar gut abgebaut wird wie natürlich vorkommende Stoffe. Unter diesen Konditionen werden 90-95\% des LAS in einer Zeitspanne von 4-5 Halbwertszeiten, also von $30-35$ Tagen, abgebaut.
Wie die weiteren Ergebnisse der Laborversuche zeigen, gilt dies jedoch nur für den Fall normaler mikrobieller Aktivität und guter Belüftung, also übertragen auf Freilandverhältnisse für Bedingungen, wie sie in der obersten Schicht des Bodens anzutreffen sind.

Unter sterilen anaeroben Verhältnissen ergeben sich unter Laborbedingungen Halbwertszeiten von bis zu 4 Monaten, sodaß die Zeit für einen $90 \%$ igen Abbau bei ca. 15 Monaten liegt.

Unter Freilandbedingungen herrschen z.B. in tieferen Bodenschichten mit geringerem Anteil an Bodenluft und eingeschränkter mikrobieller Aktivität infolge niedrigerem organischen C-Gehalts ähnliche Bedingungen wie bei dem Laborversuch, woraus vergleichbare lange Verweilzeiten abzuleiten sind. Für Flächen, auf denen jährlich Klärschlamm ausgebracht wird, könnte dies unter ungünstigen Bedingungen dazu führen, daß mit dem Klärschlamm in den Boden eingebrachtes LAS nicht vollständig abgebaut ist, bevor ein erneuter Einsatz erfolgt, sodaß daraus ein konstanter Gehalt bzw. eine Anreicherung von LAS im Boden stattfinden könnte.

Aus den in der Einleitung genannten Gründen wäre eine Verlagerung der LAS in das Grundwasser bzw. eine Mobilisierung anderer Rückstände von organischen Chemikalien möglich.

\section{Danksagung}

Für die sorgfältige Durchführung der experimentellen Arbeiten sei an dieser Stelle Frau Martina HoffMANn gedankt.

\section{Literatur}

[1] A. Marcomini; S. CAPRI; W. Giger: J. of Chromatography 403, $243-252(1987)$

[2] H. De Henau; E. MathiJs: Intern. J. Environ. Anal. Chem. 26, $279-293(1986)$

[3] U. KaWASHIMA; T. TAKENA: Yukagaku 31, 944 (1982)

[4] W. GIGER: Fresenius Z. Anal. Chem. 325-326 (1988)

[5] D. E. KILE; C. T. CHIOU: Environ. Sci. Technol. 23, 832-838 (1989)

[6] P. Gunther; W. Pestemer: Symp. Comm. of the European Communities, Cost. 681, (im Druck)

[7] M. C. Trehey; W. E. Gledhill; R. G. Orth: Anal. Chem. 62, $2581-2586(1990)$

[8] A. NaKae; K. Tsujl; M. Yamannaka: Anal. Chem. 52, 2275 (1980)

[9] K. Kawashima; T. Takena: Yukagaku 31, 944 (1982)

[10] N. LiTz; H. W. Doering; M. Thiele; H. P. Blume: Ecotox and Environ Safety 14, $103-1(6),(1987)$

[11] R. D. SwISHER: Surfactant Biodegradation. 2nd Edition, Marcel Dekker, New York 1987 pp., 218 - 224. ISBN 0-8247-6938-4

[12] C. T. JafverT: Environ. Sci. Technol. 25, 1039 - 1040 (1991)

Eingegangen: 14. 05. 1992 Akzeptiert: 09.06. 1992 